\title{
STUDY OF LEFT VENTRICULAR DIASTOLIC DIAMETER-PEAK STRESS RELATIONSHIP BEFORE AND AFTER VALVE REPLACEMENT OPERATION FOR PATIENTS WITH CHRONIC AORTIC REGURGITATION
}

\author{
Hsi-Yu Yu*,† and Jaw-Lin Wang*,† \\ *Institute of Biomedical Engineering, College of Engineering \\ and College of Medicine, National Taiwan University \\ Taipei, Taiwan \\ ${ }^{\dagger}$ Department of Surgery, National Taiwan University Hospital \\ Taipei, Taiwan \\ ${ }_{\ddagger}^{\ddagger}$ jlwang@ntu.edu.tw.
}

Accepted 31 March 2008

\begin{abstract}
Background: Patients with chronic aortic regurgitation (AR) usually have dilated left ventricle due to volume overload. Some of them will reduce in size after elimination of regurgitation, but others not. The present study evaluated the hypothesis that left ventricle end-diastolic diameter (LVEDD), left ventricle end-systolic diameter (LVESD) are related to left ventricle's peak stress $(\sigma)$ both before and after operation. Methods: Sixty-eight patients with chronic aortic regurgitation receiving valve replacement were included in the study. LVEDD, LVESD, and $\sigma$ were determined by echocardiography and cuff sphygmomanometer measurement before and beyond 6 months after operation.

Results: The results showed that LVEDD, LVESD, and $\sigma$ were decreased after the operation. In addition, $\sigma$ and LVEDD had good linear correlation (for pre-operative data, $\sigma=-3.02+0.286^{*} \mathrm{LVEDD}, R=0.556$, $P<0.001$; for post-operative data, $\left.\sigma=-11.4+0.474^{*} \operatorname{LVEDD}, R=0.736, P<0.001\right)$.

Conclusion: LVEDD and $\sigma$ had a linear relationship before and after valve replacement operation for AR patients. The higher slope in linear regression equation for post-operative $\sigma$-LVEDD relationship than that for pre-operative data may indicate improved myocardial contractile efficiency after the operation.
\end{abstract}

Keywords: Aortic regurgitation; Stress; Frank-Starling law; Left ventricle.

\section{INTRODUCTION}

The timing of aortic valve operation is crucial for patients with chronic aortic regurgitation $(\mathrm{AR})$ with left ventricular dilation to prevent the development of irreversible change of left ventricular contractile function. ${ }^{1-3}$ The intra-ventricular pressure-volume $(P V)$ curve (Fig. 1) can be derived with invasive method, the catheterization technique. The $P V$ curve is an effective tool for underlying ventricular systolic efficiency. ${ }^{4}$ The $P V$ curve is a gold standard in the evaluation of ventricular function. However, the invasiveness and cost of $P V$ catheterization limits its clinical application.

Left ventricular peak stress $(\sigma)$ and left ventricular end-systolic diameter (LVESD) ${ }^{5}$ derived from

\footnotetext{
${ }^{*}$ Corresponding author: Jaw-Lin Wang, Professor, Institute of Biomedical Engineering, College of Engineering and College of Medicine, National Taiwan University, No. 1, Sec. 1, Jen-Ai Rd., Taipei, Taiwan. Tel/Fax: +886-2-233665269; E-mail: jlwang@ntu.edu.tw.
} 


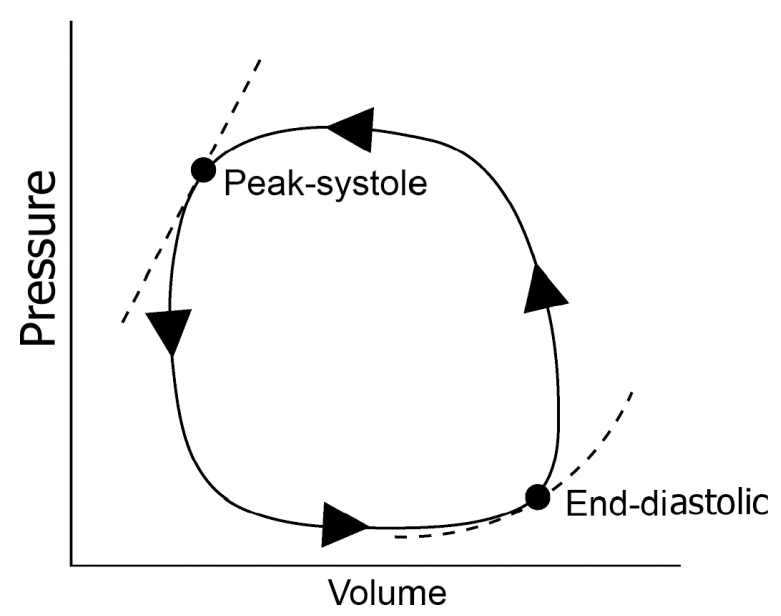

Fig. 1 Illustration of pressure-volume loop of left ventricle. Systolic action begins from right-lower corner (end-diastole) in a counterclockwise direction to the point of left-upper corner (peak-systole), which represents a state of left ventricle's maximal pumping activity. Frank-Starling law describes a stretchstress relationship between these two points.

non-invasive echocardiographic examination (see Method for detailed description) was analogous to the peak-systolic pressure and volume of $P V$ curve (Fig. 1). In addition, according to Frank-Starling law, ${ }^{6,7}$ the peak stress is also regulated by diastolic myocardial fiber length, which is a function of left ventricular enddiastolic diameter (LVEDD). The present study is to evaluate magnitude changes of peak stress, LVESD and LVEDD for AR patients before and after aortic valve replacement operation. We also want to define the linear correlation between peak stress and LVEDD both before and after operation.

\section{MATERIAL AND METHODS}

Patients with chronic aortic regurgitation receiving elective aortic valve replacement from 1993 to 2002 in one hospital (NTUH) were included in the present study. Exclusion criteria include associated diagnosis of aortic stenosis, mitral stenosis, infectve endocarditis, coronary artery disease, and incomplete echocardiographic studies. Preoperative echocardiography was performed within 3 months before operation. Measurement of systolic and diastolic parameters of left ventricle was obtained according to the recommendation of American Society of Echocardiography. ${ }^{8}$ Blood pressure was measured with cuff sphygmomanometer in supine position at the time with echocardiographic study. Operation was performed in standard open-heart method under mild hypothermic cardiopulmonary bypass and cold crystallid or blood cardioplegic arrest. All the valve replacement operations were performed with standard suturing technique under cardiopulmonary bypass support. Mechanical prostheses were either St. Jude Medical standard-cuffed, high-performance mechanical valve (St. Jude Medical Inc., St. Paul, Minneapolis 55117-9983, USA) or CarboMedics mechanical valves (CarboMedics Inc. Austin, Texas 78752-1793, USA).

Surviving patients were followed up at out-patient clinic. Echocardiography was performed 6-12 months after operation, as suggested by Bonow et al. ${ }^{2}$ Associated blood pressure was measured by cuff sphygmomanometer in supine position during the examination. Patients who were lost of follow-up were excluded from the study.

\section{Left Ventricular Wall Stress $(\sigma)$}

Deduction of Grossman's equation for $\sigma$ of left ventricle: refer to Fig. 2, where $a$ and $b$ represent the outer and inner radius of a sphere, respectively. $p_{\mathrm{i}}$ and $p_{\mathrm{o}}$ represent the pressure inside and outside the sphere, respectively. $\sigma$ represents the average circumferential wall stress

$$
\begin{aligned}
& a=\operatorname{LVEDD} / 2 \\
& b=\operatorname{LVESD} / 2 \\
& P W=(a-b) / 2=(\text { LVEDD }- \text { LVESD }) / 2 \\
& \sigma\left(b^{2}-a^{2}\right) \pi=p_{\mathrm{i}} a^{2} \pi-p_{\mathrm{o}} b^{2} \pi \\
& \sigma=\frac{p_{i} a^{2}-p_{\mathrm{o}} b^{2}}{b^{2}-a^{2}}
\end{aligned}
$$

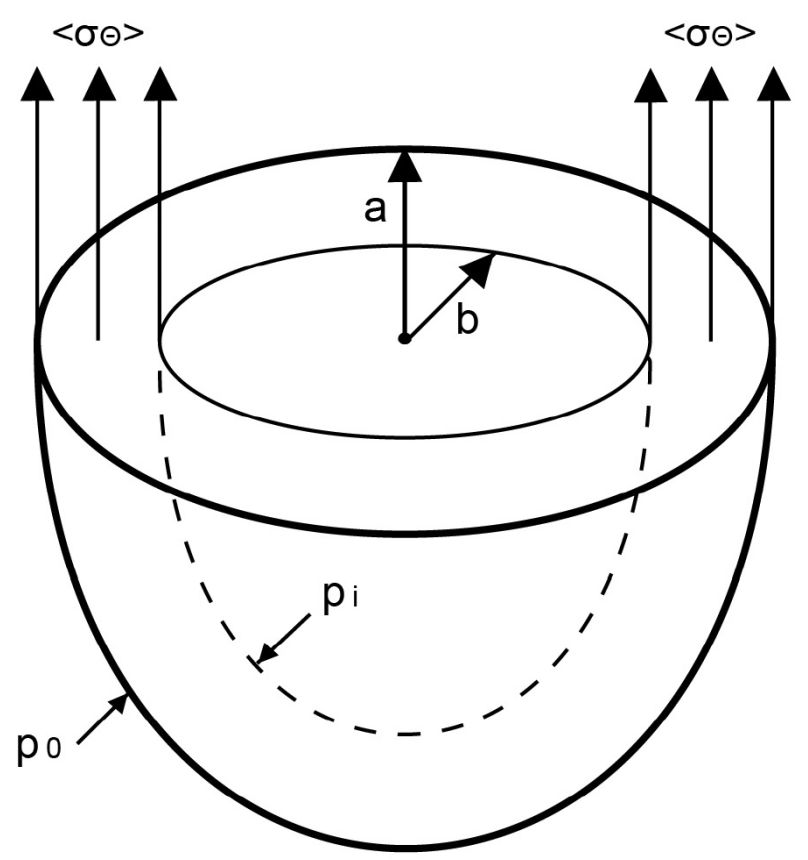

Fig. 2 Illustration for the deduction of the equation of wall stress. 
Let $p_{\mathrm{o}}=0$,

$$
\begin{aligned}
\sigma & =\frac{p_{\mathrm{i}} a^{2}}{b^{2}-a^{2}} \\
& =\frac{\mathrm{SBP} \times(\mathrm{LVESD} / 2)^{2}}{\left[(\mathrm{LVEDD} / 2)^{2}-(\mathrm{LVESD} / 2)^{2}\right]} \\
& =\frac{\mathrm{SBP} \times \mathrm{LVESD}^{2}}{(\mathrm{LVEDD}-\mathrm{LVESD}) \times(\mathrm{LVEDD}+\mathrm{LVESD})}, \\
\sigma & =\frac{\mathrm{SBP} \times \mathrm{LVESD}^{2}}{2 \mathrm{PW} \times(2 \mathrm{LVESD}+2 \mathrm{PW})} .
\end{aligned}
$$

To convert unit of $\sigma$ from $\mathrm{mmHg}$ into $\mathrm{kPa}$,

$$
\operatorname{Stress}(\sigma)=1.33 \times \frac{\mathrm{SBP} \times \operatorname{LVESD}}{4 \times \mathrm{PW} \times\left(1+\frac{\mathrm{PW}}{\mathrm{LVESD}}\right)} .
$$

1.33 is a conversion factor to express the final result in $\mathrm{kPa}$.

Peak stress $(\sigma)$ of left ventricle was calculated according to the method by Grossman et al. ${ }^{9}$

$$
\operatorname{Stress}(\mathrm{kPa})=1.33 \times \frac{\mathrm{SBP} \times \operatorname{LVESD}}{4 \times \mathrm{PW} \times\left(1+\frac{\mathrm{PW}}{\mathrm{LVESD}}\right)}
$$

for pre-operative data,

$$
\operatorname{Stress}(\mathrm{kPa})=1.33 \times \frac{(\mathrm{SBP}+\Delta P) \times \operatorname{LVESD}}{4 \times \mathrm{PW} \times\left(1+\frac{\mathrm{PW}}{\mathrm{LVESD}}\right)}
$$

for post-operative data,

where SBP is the systolic blood pressure $(\mathrm{mmHg})$, LVESD is the left ventricular end-systolic diameter $(\mathrm{mm}), \mathrm{PW}$ is the thickness of posterior wall of $\mathrm{LV}$, and $\Delta P$ is the peak pressure gradient across prosthetic aortic valve.

The pressure gradient is derived by a simplified Bernoulli's equation

$$
\Delta P=4 \times\left(V_{1}^{2}-V_{2}^{2}\right) \approx 4 \times V_{1}^{2} \text { if } V_{1} \gg V_{2} .
$$

The peak flow velocity across prosthetic valve $\left(V_{1}\right)$ is estimated by Doppler echocardiographic study.

\section{Statistics}

Analysis of pre-operative and post-operative data was performed with Chi-squared test and student- $t$ test. Linear regression analysis was applied to evaluate the relationship between $\sigma$ and LVEDD before and after the operation. For all statistical evaluations, differences in data with $P$ values of less than 0.05 were considered significantly different. All statistical work was performed using SPSS for Windows (SPSS Inc., Chicago, Illinois).

\section{RESULTS}

Sixty-eight patients were included in this study. The demographic data are listed in Table 1. The LVEDD decreased from $63.6 \pm 10.3$ to $48.1 \pm 8.0 \mathrm{~mm}$ after the operation $(P<0.001)$; LVESD decreased from $42.9 \pm$ 9.9 to $30.2 \pm 8.0 \mathrm{~mm}$ after the operation $(P<0.001)$; $\sigma$ decreased from $15.4 \pm 5.1$ to $11.4 \pm 5.3 \mathrm{kPa}$ after the operation $(P<0.001)$; LVEF increased from $61 \pm 11 \%$ to $66 \pm 13 \%$ after the operation $(P=0.018)$ (Table 2 and Fig. 3).

Figure 4 illustrates the relationship between $\sigma$ and LVEDD before and after operation. It shows a clear trend that all the plots were distributed along an imaginary linear relationship area. Linear regression analysis revealed that $\sigma=-3.02+0.286 * \mathrm{LVEDD}$ for pre-operative data, $R=0.556, P<0.001 ; \sigma=-11.4+$ $0.474 *$ LVEDD for post-operative data, $R=0.736$, $P<0.001$. Zero-stress LVEDD (value of LVEDD with $\sigma$ extrapolated to $0 \mathrm{kPa}$ ) was 10.6 and $24.1 \mathrm{~mm}$ for preoperative and post-operative data, respectively.

\section{DISCUSSION}

The present study demonstrate that $\sigma$ and LVEDD are closely related to each other before and after aortic valve replacement operation. This relationship might be a useful parameter to non-invasively evaluate the myocardial efficiency of left ventricle. In addition, higher slope between $\sigma$ and LVEDD in linear regression equation after the operation ( 0.286 for pre-operative

Table 1. Demography of Study Cases.

\begin{tabular}{lc}
\hline Variables & Values \\
\hline Gender & M 49/F 19 \\
Age (years) & $46.6 \pm 16.2$ \\
Prosthetic valve type (Xeno/SM/CM) & $2 / 30 / 36$ \\
Prosthetic valve size $(21 / 23 / 25 / 27)$ & $8 / 24 / 34 / 2$ \\
Peak pressure gradient across & $24.5 \pm 11.7$ \\
$\quad$ prosthetic valve (mmHg) & \\
\hline
\end{tabular}

Xeno: Xenograft, SM: St. Jude Mechanical valve, CM: CarboMedicus.

Table 2. Echocardiographic Parameters Before and After Operation.

\begin{tabular}{lccc}
\hline & Pre-Operative & Post-Operative & $\boldsymbol{P}$ \\
\hline LVEDD $(\mathrm{mm})$ & $63.6 \pm 10.3$ & $48.1 \pm 8.0$ & $<0.001$ \\
LVESD $(\mathrm{mm})$ & $42.9 \pm 9.9$ & $30.2 \pm 8.0$ & $<0.001$ \\
LVEF & $61 \pm 11 \%$ & $66 \pm 13 \%$ & 0.018 \\
Wall stress $(\mathrm{kPa})$ & $15.4 \pm 5.1$ & $11.4 \pm 5.3$ & $<0.001$ \\
\hline
\end{tabular}

LVEDD: Left ventricular end-diastolic diameter. LVESD: Left ventricular end-systolic diameter. LVEF: Left ventricular ejection fraction. 


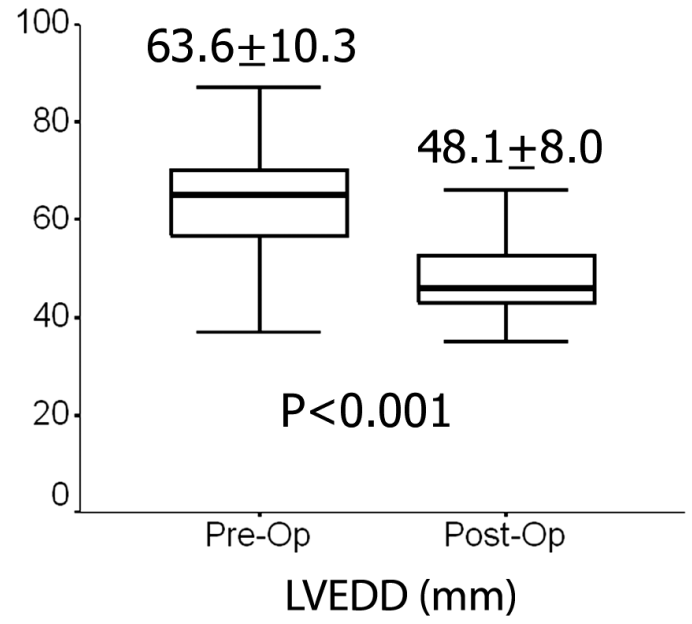

(a)

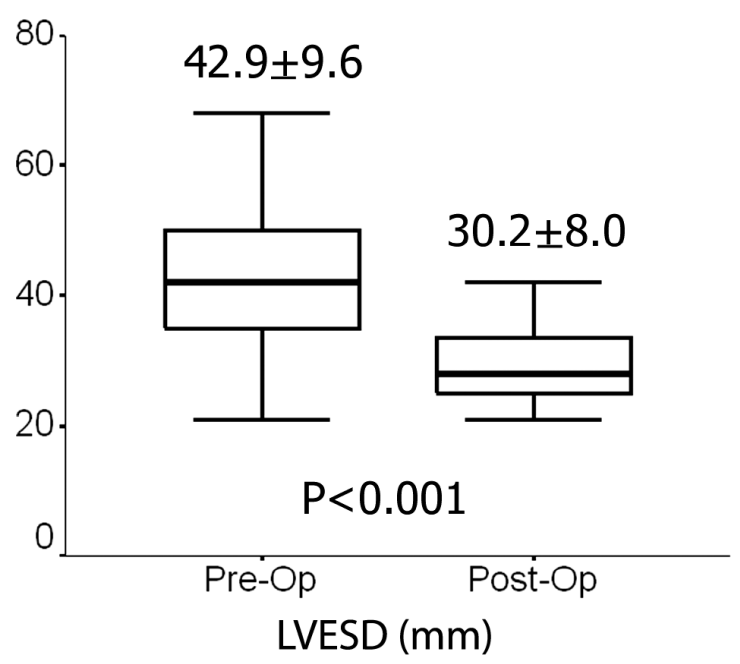

(b)

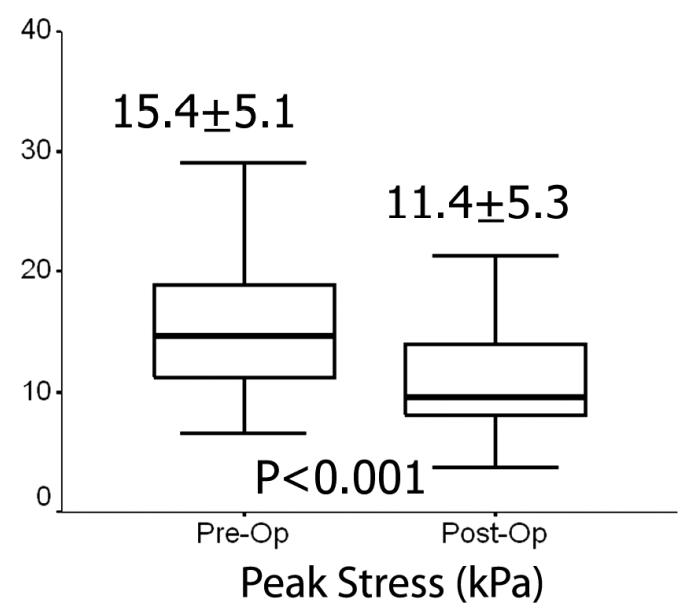

(c)

Fig. 3 Comparison of LVESD, LVEDD, and peak stress $(\sigma)$ before and after operation. It shows that LVEDD decreases from $63.6 \pm 10.3$ to $48.1 \pm 8.0 \mathrm{~mm}(P<0.001)$; LVESD decreases from $42.9 \pm 9.6$ to $30.2 \pm 8.0 \mathrm{~mm}(P<0.001) ; \sigma$ decreases from $182.8 \pm$ 50.4 to $123.1 \pm 37.4 \mathrm{kPa}(P<0.001)$.

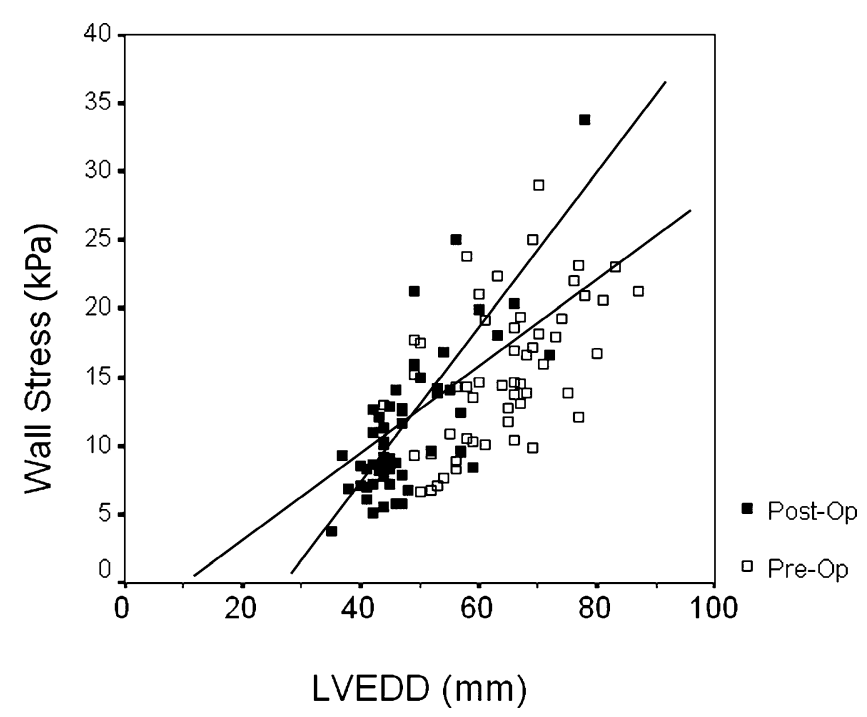

Fig. 4 Peak stresses are correlated with LVEDD for both preoperative and post-operative data. For pre-operative data, $y=$ $-3.02+0.286 x, R=0.556, P<0.001$. For post-operative data, $y=-11.4+0.474 x, R=0.736, P<0.001$. Zero-stress LVEDD (value of LVEDD with $\sigma$ extrapolated to $0 \mathrm{kPa}$ ) was 10.6 and $24.1 \mathrm{~mm}$ for pre-operative and post-operative data, respectively.

data and 0.474 for post-operative data) may indicate improved myocardial contractile efficiency after the aortic valve replacement operation for the patient group.

For cases with chronic AR, histological study has demonstrated that LV fibrosis and myocyte necrosis were in proportion to the hemodynamic burden in rabbit model even in early period. ${ }^{10}$ In another study, AR-induced myocardial damage was demonstrated to occur even before functional deterioration. ${ }^{11}$ From surgical point of view, LVESD $>55 \mathrm{~mm}$ is the most important poor prognostic indicator for LV regression after valve replacement for chronic AR..$^{2,12-14}$ But as shown in Fig. 4 in the present study, there were a number of cases with pre-operative LVESD $>55 \mathrm{~mm}$ that decreased in LV size after the operation. Our study investigated stress-length relationship for AR patients before and after the operation. It might be useful to differentiate the potential of LV regression after surgical correction of AR.

Several methods were reported in the early studies to examine contractile efficiency of left ventricle, including using systolic pressure-volume relationship ${ }^{15}$ with dobutamine stimulation, three-dimensional endsystolic maximum principal stress as estimated by MRI, ${ }^{16}$ ex vivo passive resting tension-systolic pressure relationship. ${ }^{17}$ As compared with those study methods, the present method to estimate stress-length relationship has the advantage of non-invasiveness and convenience. 
The calculation equation used in the present study to estimate LV peak stress was also used in earlier studies. ${ }^{12,18,19}$ The present study additionally evaluates myocardial contractile efficiency in a stress-length relationship. This method was more physiological than simply comparing LV peak stress.

As shown in Fig. 4, LVEDD- $\sigma$ relationship can help to differentiate ventricles with "preserved" function from "decompensated" function before operation. This differentiation is important for planning treatment strategy such as to receive valve replacement operation or heart transplantation. Second, in order to facilitate regression of LV after aortic valve operation, decrease of $\sigma$ can be achieved by using vasodilator to decrease blood pressure. Third, small-sized valve prosthesis is related to high pressure gradient, so it is disadvantageous for late $\mathrm{LV}$ regression for patients with pre-existing $\mathrm{LV}$ dilation. ${ }^{20-22}$

For most of the cases in the present study, $\sigma$ decreased after the operation, which was attributed to the elimination of aortic regurgitation, decrease in stroke volume and pulse pressure, and decrease in LVESD. But there were still some cases with increased $\sigma$ after the operation. The main reason is inadequate regression of LVESD due to pre-existing heart failure, combined with high pressure gradient across prosthetic valve, high systolic blood pressure. Therefore, to facilitate LV regression after the operation, $\sigma$ can be decreased by a number of methods, such as choosing aortic prosthesis with low-pressure gradient, using vasodilator to decrease systolic blood pressure, might be a useful way to facilitate regression of $\mathrm{LV}$ after the operation.

In conclusion, the present study demonstrates a preliminary result that $\sigma$ might provide a useful clinical parameter to estimate LV systolic efficiency. When combined with LVEDD, the stress-length relationship is a useful indicator for myocardial contractile efficiency.

\section{REFERENCES}

1. Henry WL, Bonow RO, Rosing DR, Epstein SE, Observations on the optimum time for operative intervention for aortic regurgitation. II. Serial echocardiographic evaluation of asymptomatic patients, Circulation 61(3):484-492, 1980.

2. Bonow RO, Dodd JT, Maron BJ, O'Gara PT, White GG, McIntosh CL et al., Long-term serial changes in left ventricular function and reversal of ventricular dilatation after valve replacement for chronic aortic regurgitation, Circulation 78(5 Pt 1):1108-1120, 1988.

3. Taniguchi K, Nakano S, Hirose H, Matsuda H, Shirakura R, Sakai K et al., Preoperative left ventricular function: minimal requirement for successful late results of valve replacement for aortic regurgitation, $J$ Am Coll Cardiol 10(3):510-518, 1987.

4. Suga H, Cardiac mechanics and energetics - from Emax to PVA, Front Med Biol Eng 2(1):3-22, 1990.

5. Fung YC, Biomechamics: Mechanical Properties of Living Tissues, 2nd edn., Springer-Verlag, New York, 1993.

6. Saks V, Favier R, Guzun R, Schlattner U, Wallimann T, Molecular system bioenergetics: regulation of substrate supply in response to heart energy demands, $J$ Physiol 577(Pt 3):769-777, 2006.

7. Saks V, Dzeja P, Schlattner U, Vendelin M, Terzic A, Wallimann T, Cardiac system bioenergetics: metabolic basis of the Frank-Starling law, J Physiol 571(Pt 2):253-273, 2006.

8. Sahn DJ, DeMaria A, Kisslo J, Weyman A, Recommendations regarding quantitation in M-mode echocardiography: results of a survey of echocardiographic measurements, Circulation 58(6):1072-1083, 1978.

9. Grossman W, Jones D, McLaurin LP, Wall stress and patterns of hypertrophy in the human left ventricle, $J$ Clin Invest 56(1):56-64, 1975.

10. Liu SK, Magid NR, Fox PR, Goldfine SM, Borer JS, Fibrosis, myocyte degeneration and heart failure in chronic experimental aortic regurgitation, Cardiology 90(2):101-109, 1998.

11. Lu P, Zanzonico P, Goldfine SM, Hardoff R, Magid N, Gentile R et al., Antimyosin antibody imaging in experimental aortic regurgitation, J Nucl Cardiol 4(1 Pt 1):25-32, 1997.

12. Klodas E, Enriquez-Sarano M, Tajik AJ, Mullany CJ, Bailey KR, Seward JB, Aortic regurgitation complicated by extreme left ventricular dilation: long-term outcome after surgical correction, J Am Coll Cardiol 27(3):670-677, 1996.

13. Taniguchi K, Nakano S, Kawashima Y, Sakai K, Kawamoto T, Sakaki S et al., Left ventricular ejection performance, wall stress, and contractile state in aortic regurgitation before and after aortic valve replacement, Circulation 82(3):798-807, 1990.

14. Monrad ES, Hess OM, Murakami T, Nonogi H, Corin WJ, Krayenbuehl HP, Time course of regression of left ventricular hypertrophy after aortic valve replacement, Circulation 77(6):1345-1355, 1988.

15. Grosu A, Bombardini T, Senni M, Duino V, Gori M, Picano E, End-systolic pressure/volume relationship during dobutamine stress echo: a prognostically useful non-invasive index of left ventricular contractility, Eur Heart J 26(22):2404-2412, 2005.

16. Wollmuth JR, Bree DR, Cupps BP, Krock MD, Pomerantz BJ, Pasque RP et al., Left ventricular wall stress in patients with severe aortic insufficiency with finite element analysis, Ann Thorac Surg 82(3):840-846, 2006.

17. Vahl CF, Timek T, Bonz A, Kochsiek N, Fuchs H, Schaffer L et al., Myocardial length-force relationship in end stage dilated cardiomyopathy and normal human myocardium: analysis of intact and skinned left ventricular trabeculae obtained during 11 heart transplantations, Basic Res Cardiol 92(4):261-270, 1997.

18. Bogaert J, Bosmans H, Maes A, Suetens P, Marchal G, Rademakers FE, Remote myocardial dysfunction after acute anterior myocardial infarction: impact of left 
ventricular shape on regional function: a magnetic resonance myocardial tagging study, J Am Coll Cardiol 35(6):1525-1534, 2000.

19. Verrengia M, Pacileo G, Limongelli G, Di SG, Rea A, Iacomino $\mathrm{M}$ et al., Left ventricle myocardial mechanics and textural properties in patients with Williams syndrome, J Cardiovasc Med (Hagerstown) 8(5):330-336, 2007.

20. Kulik A, Burwash IG, Kapila V, Mesana TG, Ruel M, Long-term outcomes after valve replacement for lowgradient aortic stenosis: impact of prosthesis-patient mismatch, Circulation 114(1 Suppl):I553-I558, 2006.
21. Sakamoto Y, Hashimoto K, Okuyama H, Takakura H, Ishii S, Taguchi $\mathrm{S}$ et al., Prevalence and avoidance of patient-prosthesis mismatch in aortic valve replacement in small adults, Ann Thorac Surg 81(4):13051309, 2006.

22. Tasca G, Mhagna Z, Perotti S, Centurini PB, Sabatini T, Amaducci A et al., Impact of prosthesis-patient mismatch on cardiac events and midterm mortality after aortic valve replacement in patients with pure aortic stenosis, Circulation 113(4):570-576, 2006. 